\title{
Naturaleza del Razonamiento Algebraico Elemental
}

\section{The Nature of Elementary Algebraic Reasoning}

\author{
Juan D. Godino* \\ Walter F. Castro** \\ Lilia P. Aké**** \\ Miguel R. Wilhelmi****
}

\begin{abstract}
Resumen
La introducción del razonamiento algebraico en educación primaria es un tema de interés para la investigación e innovación curricular en didáctica de las matemáticas, y presupone una visión ampliada de la naturaleza del álgebra escolar. En este trabajo proponemos una manera de concebir el razonamiento algebraico, basada en los tipos de objetos y procesos matemáticos introducidos en el enfoque ontosemiótico del conocimiento matemático. En síntesis, la consideración de una práctica matemática como algebraica se basará en la intervención de procesos de generalización y simbolización, junto con otros objetos usualmente considerados como algebraicos, tales como relaciones binarias, operaciones,

\footnotetext{
* Doctor en Matemáticas por la Universidad de Granada (UGR). Catedrático de Didáctica de la Matemática. Departamento de Didáctica de la Matemática. Universidad de Granada (UGR). Granada, España. Dirección postal: Facultad de Educación. Campus de Cartuja. 18071 Granada, España. E-mail:jgodino@ugr.es.

** Doctor en Didáctica de la Matemática por la Universidad de Granada (UGR). Profesor de la Facultad de Educación, Grupo de Investigación Matemática, Educación y Sociedad (MES) de la Universidad de Antioquia (UdeA), Medellín, Antioquia, Colombia. Dirección postal: Carrera 83 B, numero 27 A 41, Barrio Belen, Los Alpes, Medellin, Colombia. E-mail: wfcastro82@gmail.com.

*** Licenciada en Enseñanza de las Matemáticas por la Facultad de Matemáticas de la Universidad Autónoma de Yucatán (UADY), Mérida, Yucatán, México. Becaria doctoral del Programa MAECAECID en el Departamento de Didáctica de la Matemática de la Universidad de Granada (UGR), Granada, España. Dirección postal: Facultad de Educación, Campus de Cartuja, 18071 Granada, España. E. mail: lake86@gmail.com.

***** Doctor en Didáctica de la Matemática por la Universidad Pública de Navarra (UPNA). Profesor Contratado Doctor de la Universidad Pública de Navarra (UPNA), España. Dirección postal: Departamento de Matemáticas. Universidad Pública de Navarra, Campus Arrosadía s/n 31011. Pamplona, España. E-mail: miguelr.wilhelmi@unavarra.es.
} 
funciones y estructuras. Esta forma de concebir el álgebra elemental es contrastada con las caracterizaciones dadas por otros autores. Asimismo, proponemos una tipología de configuraciones algebraicas que permite definir grados de algebrización de la actividad matemática.

Palabras-clave: Álgebra Escolar. Educación Matemática. Enfoque Ontosemiótico. Configuración Algebraica. Grado de Algebrización.

\begin{abstract}
The introduction of algebraic reasoning in primary education is a subject of interest for research and curricular innovation in mathematics education, which supposes an extended vision of the nature of school algebra. In this paper we propose a way to conceive of algebraic reasoning based on the types of mathematical objects and processes introduced in the onto-semiotic approach to mathematical knowledge. In particular, considering a mathematical practice as algebraic is based on the intervention of generalization and symbolization processes, along with other objects usually considered as algebraic, such as binary relations, operations, functions and structures. This way to conceive of elementary algebra is based on and compared to the characterizations given by other authors. We also propose a typology of algebraic configurations that allows defining degrees of algebrization of mathematical activity.
\end{abstract}

Keywords: School Algebra. Mathematics Education. Onto-Semiotic Approach. Algebraic Configuration. Degree of Algebrization.

\title{
1 Soluciones aritmética y algebraica de tareas escolares
}

Un profesor propone a sus estudiantes el siguiente problema:

Problema 1: Un estudiante recibió de sus padres una cierta cantidad de dinero para comer durante 40 días. Sin embargo, encontró sitios en donde pudo ahorrar 4 euros al día en la comida. De esta forma, el presupuesto inicial le duró 60 días. ¿Cuánto dinero recibió?

Un estudiante A resolvió el problema de la siguiente manera:

Sea $D$ el dinero recibido de los padres. Representamos por $x$ el gasto diario previsto por los padres para comer 40 días: $x=D$ / 40 .

Sea $y$ el gasto diario real, que permitió comer 60 días: $y=D / 60$.

$40 x=60 y$; además $y=x-4$;

$40 x=60(x-4) ; 20 x=240 ; x=12$; Cantidad recibida: 12 X $40=480$; $480 €$.

Otro estudiante B lo resolvió de esta otra manera:

El ahorro de $4 € /$ día durante 40 días previstos supone un ahorro total de 
$160 €$. Con esta cantidad pudo comer durante 20 días más. El coste diario real fue de $160 € 20$ días $=8 €$ día. Como los días reales fueron 60, el presupuesto total será 60 días $X 8 €$ día $=480 €$.

En este ejemplo parece que habría consenso en aceptar que la solución del estudiante B se puede calificar de aritmética, mientras que la del estudiante A de algebraica. A usa letras para representar las cantidades desconocidas, y opera con ellas de acuerdo con ciertas reglas para obtener la solución. En cambio, B opera directamente con números naturales particulares a los cuales les aplica operaciones aritméticas (suma, multiplicación y división).

Sin embargo, el consenso en la consideración de una actividad como algebraica o aritmética no siempre es tan extendido. Veamos, ahora, este otro ejemplo:

Problema 2: Tres amigos, Pedro, Antonio y Pablo, no se ponen de acuerdo sobre su edad. Pedro es más viejo que Pablo; Pablo es más joven que Antonio; Antonio, a su vez, es más viejo que Pedro. ¿Quién tiene más edad?, ¿quién menos?

El estudiante B razonó de la siguiente manera:

Como Antonio es más mayor que Pedro y Pedro es mayor que Pablo entonces Antonio es también mayor que Pablo, luego Antonio es el mayor. Como Pablo es más joven que Pedro y Pedro es más joven que Antonio entonces Pablo es el más joven.

¿Sólo podemos considerar como solución aritmética aquella actividad matemática que involucra números concretos y operaciones? ¿Sólo podemos considerar como solución algebraica aquella actividad matemática que involucra el uso de incógnitas, ecuaciones, símbolos literales y operaciones con dichos símbolos, como la realizada por el estudiante A? El problema 2 y la solución dada por el estudiante $\mathrm{B}$, ¿tienen una componente esencialmente aritmética o algebraica?

Estas cuestiones no son triviales si tenemos en cuenta la abundante literatura existente donde se aborda esta problemática (CARRAHER; SCHLIEMANN, 2007; KIERAN, 2007; CAI; KNUTH, 2011); tampoco son intrascendentes desde el punto de vista educativo, ya que involucran diversas maneras de concebir la propia actividad matemática, así como su enseñanza y aprendizaje en la escuela.

\section{Problemática del álgebra escolar}

Diversas investigaciones (WAGNER; KIERAN, 1989; BEDNARZ; 
KIERAN; LEE, 1996; KIERAN, 2007; FILLOY; ROJANO; PUIG, 2008) han evidenciado las dificultades de los niños en el tránsito desde la aritmética hasta el álgebra en la escuela secundaria. Estas investigaciones han descrito aproximaciones al razonamiento algebraico que, posteriormente, permitieron aportar datos experimentales y justificaciones teóricas para apoyar la inclusión del álgebra desde la escuela primaria.

Kieran $(1989,1992)$ resalta que las dificultades de los estudiantes de secundaria en el tránsito de la aritmética al álgebra se centran en la necesidad de manipular letras y dotar a esta actividad de significado, lo que supone un cambio notable en las convenciones usadas en la aritmética y el álgebra.

El álgebra, entendida de una manera restrictiva como lenguaje simbólico, y orientada básicamente a la resolución de ecuaciones y estudio de los polinomios, aparece de manera abrupta en secundaria, sin continuidad con los temas de aritmética, medida y geometría tratados en primaria. En esta aproximación, "se atribuyen las dificultades mostradas por los estudiantes adolescentes sobre el álgebra, en gran medida, a las limitaciones de cómo se introduce la aritmética y de manera más general la matemática elemental en primaria" (CARRAHER; SCHLIEMANN, 2007, p. 675).

En razón a la dificultad del álgebra, y a que las competencias algebraicas de carácter simbólico son el resultado de un proceso de maduración más general que se desarrolla a lo largo del tiempo, se justifica que su enseñanza se inicie desde la escuela primaria (CARPENTER; FRANKLE; LEVI, 2003). En este sentido diversos investigadores han apoyado la inclusión temprana del algebra en la escuela primaria (DAVIS, 1985; VERGNAUD, 1988). Kaput (2000) hizo una propuesta, denominada algebra for all, en la que sugiere tomar acción para algebrizar el currículo de la escuela primaria con el fin de promover al álgebra como facilitadora de una mejor comprensión de las matemáticas en lugar de ser inhibidora. La inclusión del razonamiento algebraico elemental en el currículo de la escuela primaria se ha denominado Early álgebra, que, en el caso de los Principios y Estándares 2000 (NCTM, 2000), se concretó en la recomendación de incluir el contenido de álgebra desde los primeros grados.

La inclusión del álgebra en el currículo de la escuela primaria reclama una concepción más amplia del razonamiento algebraico elemental ${ }^{1}$, entendiendo

\footnotetext{
${ }^{1}$ En principio usaremos la expresión razonamiento algebraico elemental (RAE) como traducción de early algebra. No obstante, las características que proponemos para el RAE, en términos de tipos de tareas, objetos y procesos algebraicos implicados, permiten incluir en esta noción el álgebra de secundaria, reforzando, de esta manera, una visión integrada del álgebra escolar. En este trabajo consideramos las expresiones razonamiento algebraico y pensamiento algebraico como equivalentes, aunque algunos autores establecen distinciones entre las nociones cognitivas correspondientes.
} 
que dicho razonamiento se puede poner de manifiesto no sólo en tareas relacionadas con la aritmética, la medida, la geometría o con el análisis de datos, sino que lo hace con diversos grados de algebrización. La presencia de los objetos y procesos reconocidos como algebraicos es gradual, sistemática y progresiva.

El enfoque del early algebra tiene consecuencias epistémicas y didácticas. Epistémicamente la inclusión del álgebra en la escuela elemental supone un cambio del foco de atención desde los aspectos simbólicos y procedimentales hacia aspectos estructurales del razonamiento algebraico. Carraher y Schliemann (2007) afirman que la mayoría de los autores han trabajado sobre dimensiones específicas de interés y que relativamente pocos han tratado de caracterizar el campo de manera exhaustiva.

Cuando lo han intentado, la estructura categórica ocasionalmente exhibe inconsistencias y solapamientos. Por ejemplo, el desglose del álgebra en generalización, resolución de problemas, modelización, y funciones, mezcla procesos de razonamiento no disjuntos (generalización y resolución de problemas) con tópicos de matemáticas (funciones) y otro (modelización) (Bednarz, 1996) que puede ser entendido, bien como tema matemático o un conjunto de procesos de razonamiento. (CARRAHER; SCHLIEMANN, 2007, p. 676).

Esta observación lleva a los autores citados a considerar que, posiblemente, el análisis del pensamiento algebraico está todavía en su infancia.

Este nuevo enfoque, basado en los aspectos estructurales, necesita no sólo una descripción y fundamentación, sino la determinación de medios para abordar los problemas de aprendizaje y de enseñanza relacionados con las nuevas tareas y competencias algebraicas, así como acciones concretas para la formación de profesores en este campo. En definitiva, se establece un programa de investigación específico en 4 etapas:

1. Análisis previo: ¿cuándo una actividad se puede considerar que contiene un componente algebraico esencial?

2. Didáctica descriptiva: ¿bajo que condiciones se construyen y comunican conocimientos relacionados con esta nueva concepción del álgebra?

3. Didáctica normativa: ¿qué condiciones se requieren para la enseñanza y aprendizaje basada en la nueva concepción?

4. Formación de profesores: ¿qué formación inicial y continua es 
necesaria para que maestros de primaria y profesores de secundaria puedan implementar la nueva visión del álgebra?

En este trabajo nos centramos en el punto primero. Avanzar en la clarificación de la naturaleza del razonamiento algebraico elemental es un tema complejo, pero necesario desde el punto de vista educativo. Como afirma Radford (2000, p. 238), "necesitamos profundizar en nuestra propia comprensión de la naturaleza del pensamiento algebraico y la manera en que se relaciona con la generalización". La elaboración de un modelo comprensivo puede ayudar a articular coherentemente el currículo matemático escolar con los distintos niveles escolares, y facilitar el diseño de actividades instruccionales que favorezcan el surgimiento y consolidación progresivos del razonamiento algebraico.

En este trabajo abordaremos este problema, utilizando algunas herramientas teóricas del enfoque ontosemiótico del conocimiento matemático (GODINO, 2002; GODINO; BATANERO; FONT, 2007). Consideramos, junto con diversos autores (MASON, 1996; MASON; PIMM, 1984; CARRAHER; MARTÍNEZ; SCHLIEMANN, 2008; COOPER; WARREN, 2008), que la generalización es un rasgo característico del razonamiento algebraico, así como los medios para simbolizar, tanto las situaciones de generalización, como las de indeterminación (uso de incógnitas y ecuaciones para modelizar situaciones). Asimismo, las nociones de relación, operación y estructura son propias del álgebra. En la siguiente sección analizamos, brevemente, estos rasgos característicos del álgebra. Posteriormente, presentamos una visión integrada sobre el razonamiento algebraico elemental, que considera los rasgos característicos del álgebra destacados por otros autores, y que permite reconocer distintos tipos y grados de algebrización de la actividad matemática.

\section{Rasgos característicos del álgebra escolar}

Diversos autores se han interesado por reflexionar acerca de los rasgos que caracterizan el álgebra escolar. Kieran (2007), apoyándose en propuestas de diversos autores, elabora un modelo que sintetiza las actividades del álgebra escolar en tres tipos: generacional, transformacional y global o de meta-nivel. Las actividades de tipo generacional implican la formación de expresiones y ecuaciones, las cuales considera como los objetos del álgebra. Incluye en esta categoría como ejemplos típicos, a) ecuaciones que contienen una incógnita que representan situaciones problema, b) expresiones de generalidad que surgen de patrones geométricos o secuencias numéricas, c) expresiones de reglas que 
gobiernan relaciones numéricas.

Las actividades de tipo transformacional (o actividades basadas en reglas), incluyen, por ejemplo, agrupar términos semejantes, factorizar, desarrollar, sustituir una expresión por otra, sumar y multiplicar expresiones polinómicas, resolver ecuaciones e inecuaciones, simplificar expresiones, sustituir valores numéricos en expresiones, trabajar con ecuaciones y expresiones equivalentes etc. Aunque la mayor parte de estas actividades se interesan por los cambios en la forma simbólica de una expresión o ecuación que mantienen la equivalencia, esto no implica que se trate de actividades rutinarias ya que su justificación implica la aplicación de axiomas y propiedades de las estructuras correspondientes.

La tercera categoría de actividades propuesta por Kieran (2007, p.714) y denominada global/o de nivel meta, sugiere el uso de procesos matemáticos más generales. Son actividades para las que el álgebra se usa como una herramienta, pero que no son exclusivas del álgebra. En concreto, se incluye en esta categoría la resolución de problemas, modelización, estudio de patrones generalizables, justificar y probar, formular predicciones y conjeturas, estudiar el cambio en situaciones funcionales, buscar relaciones o estructura etc. "actividades que se pueden ciertamente realizar sin usar expresiones simbólicoliterales algebraicas".

Parece que hay consenso en que uno de los rasgos característicos del razonamiento algebraico es su manera de abordar los procesos de generalización matemática, esto es, el estudio de situaciones en las que se pasa de considerar casos particulares de situaciones, conceptos, procedimientos etc., (objetos determinados) a las clases o tipos de tales objetos.

Dörfler (1991, p. 84) equipara abstracción con generalización, y, esta última, la vincula con el uso de variables, rasgo característico del álgebra. Distingue entre generalizaciones empíricas y teóricas. Las generalizaciones empíricas se basan en el reconocimiento de características o cualidades comunes a los objetos o situaciones, mientras que las teóricas se derivan de la identificación de invariantes esenciales en sistemas de acción (materiales o mentales), así como en las condiciones de realización o los resultados de dichas acciones. "Las generalizaciones teóricas no tienen sus raíces exclusivamente en las propias cosas sino en la creación, transformación y actividad operativa, en las acciones de los seres humanos". El papel esencial que tienen los símbolos en los procesos de generalización, como variables referenciales de los elementos que intervienen en la acción y las relaciones entre ellos, lleva a Dörfler (1991, p.84) a concluir 
que "generalizar significa construir variables".

Según Kieran (1989, p, 165), "para una caracterización significativa del pensamiento algebraico no es suficiente ver lo general en lo particular, se debe ser capaz de expresarlo algebraicamente". Esa expresión es una condición previa para la manipulación de las representaciones simbólicas que produce otras equivalentes más útiles para la resolución de los problemas.

Sin embargo, una tendencia reciente entre los investigadores propone separar el simbolismo algebraico del pensamiento algebraico. "Esta consideración separada es impulsada por dos factores: (1) el reconocimiento de la posibilidad de manipulación simbólica sin sentido, y (2) la tendencia en la escuela elemental de introducir el 'álgebra temprana', esto es, focalizar la atención en la estructura más bien que en el cálculo" (ZAZKIS; LILJEDAHL, 2002, p. 398). En la perspectiva del álgebra temprana, el reconocimiento de lo general desempeña un papel esencial como condición previa de la expresión. Kaput y Blanton (2001) ven la generalización y la expresión sistemática progresiva de la generalidad como subyacente a todo el trabajo que hacemos en álgebra.

El simbolismo algebraico es el lenguaje que da voz al pensamiento algebraico, "el lenguaje que expresa la generalidad" (MASON, 1996). Pero la naturaleza de dicho lenguaje puede ser diversa. Hay un desfase entre la habilidad de los estudiantes para reconocer y expresar verbalmente un cierto grado de generalidad y la habilidad para emplear la notación algebraica con facilidad. English y Warren (1998) consideran que la parte más difícil es expresar algebraicamente las generalizaciones.

En este sentido Radford (2003), al estudiar los tipos de generalización de patrones numérico-geométricos por estudiantes de secundaria, identifica la puesta en funcionamiento por dichos estudiantes de dos tipos de generalización pre-algebraica: la generalización factual y la generalización contextual. En el primer tipo, se trata de una generalización de acciones en la forma de un esquema operacional, esquema que permanece ligado al nivel concreto de uso de los símbolos numéricos, a términos deícticos y gestos, como medios semióticos de objetivación; lo general o lo indeterminado quedan sin nombrar. Las generalizaciones contextuales suponen un nivel más avanzado, sin alcanzar el nivel de las generalizaciones simbólicas algebraicas; en este caso se generalizan no solo las acciones numéricas sino también los objetos y las acciones. "Van más allá del dominio de las figuras específicas y tratan con objetos genéricos (como la figura) que no pueden ser percibidos por nuestros sentidos" (RADFORD, 2003, p. 65). 
Con ser esencial para el álgebra, la generalización no se estudia exclusivamente de manera algebraica, ni todas las actividades algebraicas involucran generalización.

Algunos autores relacionan el álgebra con el tratamiento de objetos de naturaleza indeterminada, tales como incógnitas, variables y parámetros. "Lo que esto significa es que, en álgebra, se calcula con cantidades indeterminadas (esto es, se suma, resta, divide, etc., incógnitas y parámetros como si se conocieran, como si fueran números específicos)" (RADFORD, 2010, p. 2). En el problema 1 de la introducción hay que hallar una cantidad de dinero recibido de los padres, que es un valor particular específico, pero no determinado inicialmente. El gasto diario previsto y el real también son valores específicos indeterminados en los datos del problema. La técnica algebraica característica es nombrar tales cantidades indeterminadas y operar con ellas como si fueran conocidas. Se trata de una práctica típicamente algebraica que no involucra procesos de generalización.

Otro rasgo característico del álgebra es el estudio de las relaciones de equivalencia y sus propiedades, así como el estudio de las operaciones entre los elementos de los conjuntos numéricos, o de otro tipo, y las propiedades de las estructuras que se generan en los mismos. En relación con el pensamiento relacional, la investigación sobre álgebra temprana se ha interesado, particularmente, por indagar la comprensión de los estudiantes de los significados operacional y relacional del signo igual, esto es, la distinción entre el uso del signo igual para indicar el resultado de operaciones, o la equivalencia de dos expresiones (CARPENTER et al., 2005; STEPHENS, 2006; MOLINA; CASTRO; CASTRO, 2009).

De las descripciones del pensamiento algebraico y de la actividad algebraica, se puede concluir que la consideración de una actividad como algebraica tiene contornos difusos. En algunos casos, puede haber un claro consenso, como en las actividades generacionales y transformacionales formación y manipulación de expresiones simbólico-literales -, pero no así en otras actividades como modelización, resolución de problemas, o con actividades típicas del early algebra, como las equivalencias de expresiones aritméticas. Parece pertinente considerar que en el proceso de transición desde la aritmética hasta el álgebra cruza una zona transicional en la que se admite que las tareas matemáticas pueden exhibir objetos y procesos algebraicos con una presencia gradual, pero creciente. 


\section{Aproximación al álgebra desde un enfoque ontosemiótico}

La perspectiva pragmatista, antropológica y semiótica del enfoque ontosemiótico del conocimiento matemático (EOS) (GODINO, 2002; GODINO; BATANERO; FONT, 2007) aporta herramientas teóricas para analizar la actividad matemática en general y, en particular, para el tipo de actividad que caracteriza el álgebra, como vamos a mostrar a continuación. El EOS permite caracterizar el álgebra en términos de los tipos de objetos y procesos que intervienen en la práctica matemática.

La actividad algebraica tiene lugar cuando una persona aborda la solución de cierto tipo de problemas o tareas, realizando determinadas prácticas operativas y discursivas. En dichas prácticas intervienen elementos de naturaleza diversa, en particular, medios de expresión, reglas conceptuales, procedimentales, proposiciones y justificaciones. En consecuencia, la caracterización de una práctica, y el pensamiento que la acompaña, como de índole algebraica habrá que hacerla en términos de la presencia de los tipos de objetos y de procesos que intervienen en la misma. Dichos objetos y procesos vinculados a las prácticas, están interrelacionados formando configuraciones.

\subsection{Tipos de objetos algebraicos primarios}

En el EOS se propone una tipología de objetos que intervienen y emergen de las prácticas matemáticas, entendiéndose por práctica "toda actuación o expresión (verbal, gráfica, etc.) realizada por una persona (o compartidas en el seno de una institución) para resolver problemas matemáticos, comunicar a otros la solución obtenida, validarla o generalizarla a otros contextos y problemas" (GODINO; BATANERO, 1994, p. 334).

La figura 1 resume los seis tipos de objetos primarios, y vamos a utilizarlos como pauta para indagar los tipos de objetos algebraicos.

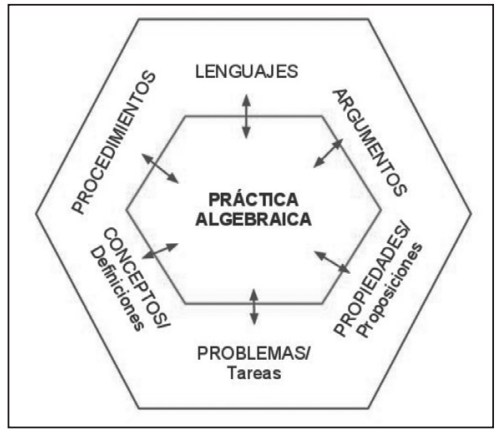

Figura 1 - Objetos implicados en la práctica algebraica 
La consideración de una práctica matemática como de índole algebraica puede hacerse con base en la presencia de cierto tipo de objetos, usualmente considerados en la literatura como algebraicos. Estos pueden ser conceptos, procedimientos, propiedades, argumentos, expresados preferentemente con un lenguaje alfanumérico. En una primera aproximación, vamos a considerar como tipos de objetos algebraicos primarios los siguientes:

1) Relaciones binarias - de equivalencia o de orden - y sus respectivas propiedades (reflexiva, simétrica, transitiva; antisimétrica etc.)

2) Operaciones y sus propiedades, realizadas sobre los elementos de conjuntos de objetos diversos (números, transformaciones geométricas etc.). El denominado cálculo algebraico se caracteriza por la aplicación de propiedades tales como asociativa, conmutativa, distributiva, existencia de elemento neutro y de un inverso. Pueden intervenir, también, otros conceptos tales como ecuación, inecuación, incógnita, así como procedimientos tales como eliminación, trasposición de términos, factorización, desarrollo de términos, entre otros.

3) Funciones, sus tipos, operaciones con funciones, y propiedades; funciones proposicionales (verdadero/falso); variables, fórmulas, parámetros.

4) Estructuras y sus tipos (semigrupo, monoide, semimódulo, grupo, módulo, anillo, cuerpo, espacio vectorial etc.) propias del álgebra superior o abstracta.

Estos tipos de objetos algebraicos básicos se pueden expresar con diversos lenguajes, preferentemente de tipo alfanumérico, si nos atenemos al sentido clásico del álgebra que describe Kieran (1989, p, 165). Pero, en el contexto escolar, también se usan otros medios de expresión, en particular el lenguaje ordinario, gráfico, tabular, incluso gestual (RADFORD, 2003; ARZARELLO, 2006). Un tipo de actividad algebraica primaria será la traducción, o transformación entre distintos lenguajes (registros de representación), particularmente la conversión (DUVAL, 2008) entre el registro de la lengua natural al registro alfanumérico.

\subsection{Relatividad contextual de las prácticas algebraicas}

En el marco del EOS las prácticas matemáticas y los objetos que intervienen en las mismas se pueden contemplar desde distintos puntos de vista, según el contexto o el juego de lenguaje en que tienen lugar dichas prácticas. La figura 2 resume dichos puntos de vista, representados como pares de dualidades para indicar las relaciones dialécticas que se establecen entre las mismas. 


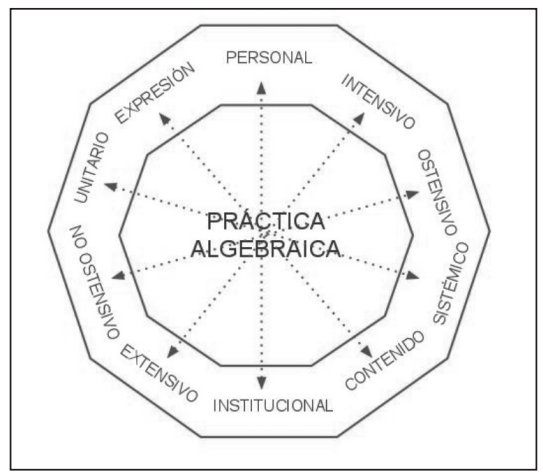

Figura 2 - Relatividad contextual de la práctica algebraica

En el caso de las prácticas algebraicas la dualidad extensivo - intensivo (particular - general), y los procesos asociados de particularización-generalización, tiene una importancia especial, dado el papel de la generalización como uno de los rasgos característicos del álgebra, según hemos visto en la sección anterior.

Un objeto se dice que es extensivo si interviene en una práctica matemática como un ejemplar particular; mientras, se dice que es intensivo si interviene como un tipo, clase o generalidad. Estos atributos de los objetos matemáticos, emergentes de los procesos duales de particularización y generalización, son relativos al juego de lenguaje en que participan, y no son entidades absolutas. Por ejemplo, en el estudio de las funciones, $y=2 x+1$, sería una función particular perteneciente a la clase o tipo de funciones lineales, $y=$ $m x+n$; esta última expresión será un objeto intensivo. No obstante, en el estudio de las funciones polinómicas, la función lineal, $y=m x+n$, será un caso particular (un extensivo) de dicha clase de funciones (un intensivo).

La función lineal particular, $y=2 x+1$, está constituida por otros extensivos, los números 2, 1, la operación de sumar números reales, así como de otros intensivos, tales como es el conjunto $R$ de números reales sobre el que toma valores la variable independiente $x$ y la dependiente $y$ de dicha función. Asimismo, al pedir a los alumnos que continúen la serie de números, 1, 3, 5, 7, 9, ..., y que encuentren la ley general que siguen, esperamos que digan algo así como $2 x+1$. Los números particulares $1,3,5, \ldots$ son objetos extensivos, mientras que la regla general, y la serie completa de números impares, resultado del proceso de generalización, son objetos de naturaleza intensional.

Esta manera de abordar el estudio de la generalización (y el proceso dual de particularización) muestra claramente el carácter relativo y contextual 
de tales procesos, así como la existencia de distintos niveles o grados de generalización. De la misma manera que los elementos de un conjunto pueden ser otros conjuntos, los objetos intensivos pueden dar lugar a nuevos objetos intensivos de mayor generalidad.

La creación de objetos intensivos está íntimamente relacionada y dependiente de otro proceso primario, como es el de simbolización o representación. En el estudio de la función $y=2 x+1$, el conjunto de los números reales $\mathrm{R}$ está representado (aquí de manera tácita) por las letras $x$ e $y$, las cuales se consideran como variables que toman valores en $\mathrm{R}$. Dado que $\mathrm{R}$ es un conjunto estructurado con unas operaciones que cumplen determinadas propiedades, la expresión simbólica, $2 x+1$, interpretable en el cuerpo algebraico $\mathrm{R}$, ha producido un objeto de un nuevo orden de generalidad que es la función lineal.

La dualidad unitario - sistémico permite describir los procesos mediante los cuales una entidad compuesta o sistémica (un intensivo) pasa a ser vista como una entidad unitaria (proceso de reificación, entificación, objetivación). Una vez que un intensivo es visto como una entidad unitaria podrá participar en otros procesos de generalización y dar lugar a intensivos de orden superior.

Asimismo, la dualidad ostensivo - no ostensivo aporta una nueva comprensión de los procesos de generalización, a los objetos intensivos resultantes, y a los artefactos que, necesariamente, deben intervenir para que tenga lugar la generalización. Con la ostensión nos referimos a los medios semióticos de objetivación (RADFORD, 2003), a los recursos perceptivos de expresión (simbólicos, o de cualquier otro tipo). Usualmente los objetos matemáticos (conceptos, proposiciones, ...) se consideran objetos ideales o mentales, o sea, objetos no ostensivos. Sin embargo, su producción y comunicación debe hacerse con la intervención de objetos perceptibles (palabras, símbolos, gestos,...), esto es, objetos ostensivos. Las generalidades o abstracciones, sean conceptos, procedimientos, propiedades, son en sí mismas no ostensivas, pero su manipulación por el sujeto requiere el uso de símbolos ostensivos.

La complejidad del aprendizaje de la matemática, esencialmente caracterizada por la presencia de procesos de generalización y entidades generales (intensivos), se puede comprender si tenemos en cuenta la intervención conjunta de procesos de idealización, discriminando el intensivo de sus posibles materializaciones o representaciones, y procesos de reificación. El resultado final de la generalización es un nuevo objeto cuya naturaleza es diferente de los componentes de donde proviene. 


\section{Tipos de configuraciones algebraicas}

Los objetos y procesos que hemos descrito en la sección anterior aportan criterios para distinguir distintos tipos de configuraciones algebraicas, las cuales permitirán discriminar diferentes tipos y grados de algebrización de la actividad matemática. Habrá tareas matemáticas que pongan en juego, de manera específica, relaciones binarias, operaciones, funciones, estructuras, sugiriendo la definición de configuraciones de tipo relacional, operacional, funcional, estructural. También habrá tareas cuyo foco de atención será la transformación entre distintos modos de expresión, particularmente entre los lenguajes natural, icónico, gestual etc., a lenguaje alfanumérico (configuración de tipo transformacional).

La consideración de los procesos de particularización - generalización, y los objetos que se generan en los mismos (extensivos, intensivos), o que intervienen o se aplican en los mismos, aporta un nuevo criterio de clasificación de las prácticas algebraicas, las configuraciones de objetos y el pensamiento que las acompaña. La presencia de objetos intensivos en una práctica matemática nos sirve para reconocer indicios de un cierto nivel de abstracción o generalización. La emergencia de los objetos intensivos atraviesa por distintos momentos o etapas, cada una de las cuales le aporta distintos niveles o capas de generalidad. Un número, 3, una figura geométrica, el triángulo, se presenta como entidad unitaria, ideal, abstracta, general; pero, al mismo tiempo, su construcción, idealización, abstracción, reificación, pasa por distintos momentos y contextos, cada uno de los cuales le impregna de significados parciales y distintos niveles de generalidad.

La presencia de objetos intensivos (generalidades, conceptualizaciones, abstracciones), en alguno de sus niveles o capas de generalidad, será un rasgo característico de actividad algebraica elemental. Entendida el álgebra de esta manera, supone ampliar su presencia en las matemáticas escolares, ya que en las primeras actividades matemáticas, como pueden ser las de conteo de colecciones de objetos, realizados por niños de preescolar, hay procesos de generalización - conceptualización.

El razonamiento algebraico se inicia a partir de las actividades aritméticas de cuantificación de cantidades mediante los procesos de simbolización numérica. Los símbolos numéricos se organizan, desde los primeros niveles, como un sistema formado por elementos relacionados mediante ciertas operaciones; tales operaciones, que inicialmente refieren a acciones sobre cantidades, pasan a ser 
operaciones sobre los propios símbolos y vienen relacionadas con un sistema de propiedades estructurales. Se obtiene, de este modo, un primer ejemplo de estructura algebraica: los semigrupos aditivo y multiplicativo de los números naturales. Ciertamente que los niños no van a estudiar estas estructuras algebraicas como tales, pero en el trabajo con las operaciones aritméticas (que son también un tipo de funciones) ponen en juego conceptos y teoremas en acto, en el sentido descrito por Vergnaud (1990), que son propios de las mencionadas estructuras.

El surgimiento del razonamiento algebraico se basa en un primer proceso de generalización: de la cantidad de una magnitud concreta (por ejemplo, número de canicas) se pasa al símbolo que representa una cantidad de una magnitud cualquiera (número de personas, caramelos etc.). El sistema de símbolos emergentes de este sistema de prácticas de cuantificación y ordenación, regulado mediante los axiomas de Peano, se convierte en el sistema numérico natural.

La idea clave detrás de esta nueva visión es que la aritmética es parte del álgebra, esto es, la parte que trata con los sistemas numéricos, la recta numérica, funciones simples, etc. La aritmética trata con la parte del álgebra en la que números particulares y medidas son tratadas como ejemplos de otros ejemplos más generales. (CARRAHER; SCHLIEMANN, 2007, p. 698).

Un nivel más avanzado de pensamiento algebraico se pone de manifiesto en las actividades que involucran relaciones binarias y correspondencias (funciones), primero entre cantidades, entre símbolos estructurados, después. La igualdad, como relación de equivalencia entre números (y como indicación del resultado de una acción-operación) es otro objeto emergente de la práctica matemática que caracteriza el razonamiento algebraico. A partir de la igualdad como relación de equivalencia se obtienen clases de equivalencia y conjuntos cocientes, objetos característicos del álgebra; a partir de las correspondencias (aplicaciones, funciones) se obtienen los isomorfismos entre estructuras etc.

Un criterio adicional de clasificación de las configuraciones algebraicas se deriva del hecho de que las prácticas matemáticas pueden ser orientadas hacia la generación de nuevos objetos intensivos (prácticas generativas), o simplemente hacia la aplicación de objetos intensivos (prácticas de aplicación, modelización).

La figura 3 resume los criterios o variables que describen los tipos de 
configuraciones algebraicas presentes en la actividad matemática, de acuerdo con el análisis que acabamos de presentar. La aplicación sistemática de este esquema da lugar a una tipología de configuraciones algebraicas. En el nivel más básico o primario de algebrización estará la configuración intensional (aplicativa o generativa), expresada con lenguaje natural, icónico, gestual. El uso de lenguaje alfanumérico, junto con objetos algebraicos de tipo relacional, operacional y estructural caracterizará niveles más avanzados de algebrización.

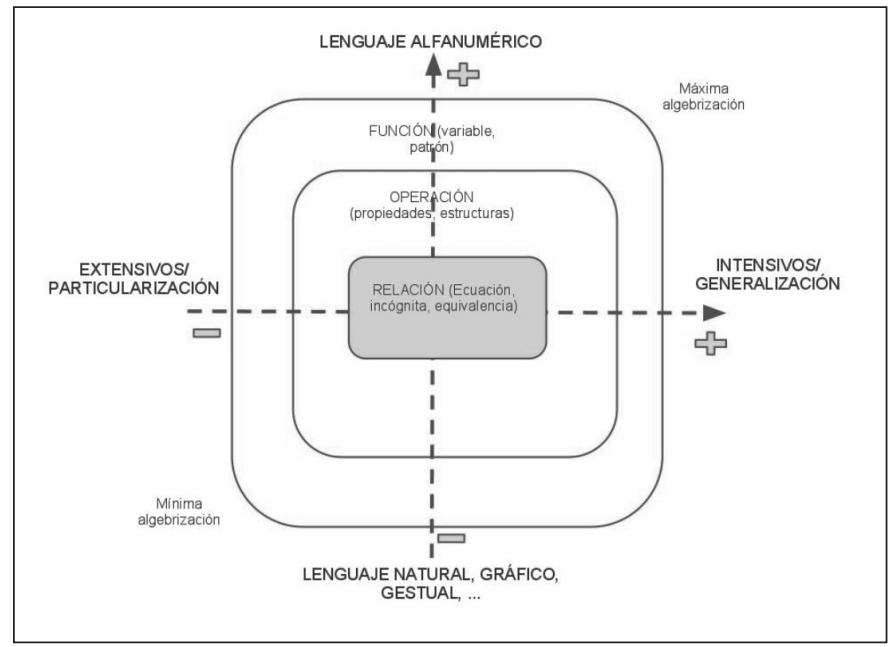

Figura 3 - Variables que caracterizan la actividad algebraica

Las actividades usadas en las investigaciones sobre pensamiento relacional (CARPENTER; FRANKLE; LEVI, 2003; STEPHENS, 2006) son de tipo mixto, relacional-operacional ya que en ellas intervienen secuencias de operaciones aritméticas combinadas con el signo igual en su acepción de relación de equivalencia, y requieren la aplicación de propiedades de las operaciones aritméticas (asociativa, conmutativa, distributiva,...). Además, en su mayoría son de tipo aplicativo y expresadas con lenguaje simbólico - aritmético.

La manera de concebir el álgebra (y de manera equivalente, el pensamiento/ razonamiento algebraico) que hemos descrito postula una cierta imbricación y continuidad entre el álgebra y el resto de contenidos matemáticos (aritmética, medida, geometría, análisis, estocástica). Siempre que reconozcamos la presencia de objetos intensivos en una práctica matemática, en alguno de sus niveles de generalidad o intensión, estamos en condiciones de atribuir un cierto grado de algebrización a dicha práctica, tanto si el intensivo se expresa de manera 
alfanumérica, como si no.

Sin embargo, dado que el uso de representaciones alfanuméricas para los intensivos que intervienen en una práctica matemática facilita la reflexión sobre los mismos, y el acceso a nuevos niveles de generalidad y cálculo operatorio, parece conveniente considerar a dichas configuraciones como algebraicas en sentido estricto, y aquellas en que no se usan dichas representaciones como configuraciones protoalgebraicas. Esto no debe suponer el abandono de la hipótesis de continuidad, ya que no hay álgebra sin protoálgebra² ${ }^{2}$.

Ontogénicamente hablando, hay espacio para una amplia zona conceptual donde los estudiantes pueden comenzar a pensar algebraicamente, aunque no recurran aún (o al menos no en gran medida) a signos alfanuméricos. Esta zona, que podemos llamar la zona de emergencia del pensamiento algebraico, ha permanecido largamente ignorada, como resultado de nuestra obsesión con reconocer el álgebra solo en lo simbólico. (RADFORD, 2010, p. 3).

Bolea, Bosch y Gascón (2001) reconocen que las organizaciones matemáticas pueden tener un carácter más o menos algebrizado, y caracterizan el álgebra escolar como un instrumento genérico de modelización de la actividad matemática. Nuestro análisis de los tipos y grados de algebrización de las tareas matemáticas difiere sustancialmente del realizado por estos autores, que establecen una frontera objetiva, externa al sujeto que realiza la actividad, y determinada epistemológicamente a priori. Sin embargo, según el EOS, la frontera aritmética-álgebra tiene una naturaleza contextual y funcional, de tal manera que su descripción afecta, necesariamente, a las diversas dimensiones de la realidad didáctica, no exclusivamente a la epistemológica.

Kaput, Carraher y Blanton (2008, p. xxi) se preguntan si el álgebra es lo mismo que la generalización, y, si así fuese, ¿no estaría en todas partes? Una visión inclusiva del razonamiento algebraico hace difícil distinguir entre pensar algebraicamente y pensar matemáticamente (o simplemente) pensar. "Ciertamente, los educadores matemáticos necesitan ser claros sobre a qué se refieren con pensamiento algebraico en los casos en los que los estudiantes no usan notación algebraica".

\footnotetext{
${ }^{2}$ Usamos el término protoálgebra para designar el sentido ampliado del álgebra que venimos describiendo en este trabajo, esto es, a la actividad matemática que pone en juego configuraciones intensionales en cualquier nivel de generalidad y simbolización. Este uso difiere del sentido histórico atribuido por Puig (2009), y del objeto protomatemático introducido en la teoría de la transposición didáctica de Chevallard (1991).
} 
Radford (2011, p. 308) estudia los límites entre el pensamiento aritmético y algebraico al reconocer que, "la generalidad es un rasgo típico general de la cognición humana y animal y puede ser de diversa naturaleza - aritmética, geométrica u otra". En el contexto de experiencias de reconocimiento de patrones numéricos - geométricos, por niños de $2^{\circ}$ grado, este autor encuentra que en ciertos momentos los niños son capaces de reconocer una característica común en determinadas figuras de una secuencia figural; pero este proceso no lo considera de índole algebraica, sino que forma parte de un proceso de formación de conceptos, accesible también a otras especies (aunque dentro de ciertos límites). El pensamiento algebraico no se puede restringir al uso de letras, ni tampoco ampliar al uso de generalizaciones conceptuales, pues:

lo que distingue el pensamiento aritmético del algebraico es el hecho de que en este último se tratan cantidades indeterminadas de una manera analítica. En otras palabras, consideras cantidades indeterminadas (p.e. incógnitas o variables) como si fueran conocidas y realizas cálculos con ellas como lo haces con números conocidos. (RADFORD, 2011, p. 318).

La indeterminación y el carácter analítico están ligados en un esquema o regla que permite a los estudiantes tratar con cualquier figura de la secuencia, cualquiera que sea su tamaño. Es una regla ejemplificada en casos particulares (p. e. 12 más 12, más 1), donde los números son tratados no como meros números, sino como constituyentes de algo más general.

Así, en el EOS se interpreta la frontera entre la aritmética y el álgebra, señalada por Radford y otros autores, en términos de la dualidad intensivoextensivo. Puesto que esta dualidad es relativa a un contexto donde se desarrolla una práctica algebraica, la frontera no es estable ni está objetivamente establecida; de hecho, está esencialmente ligada al reconocimiento, por el sujeto que realiza la actividad, de las reglas que conforman los objetos intensivos que intervienen, así como por su expresión mediante cualquier registro semiótico y posterior tratamiento analítico.

\section{Tipos y grados de algebrización de tareas escolares}

Describimos, a continuación, ejemplos de tareas matemáticas analizadas usando el modelo de razonamiento algebraico descrito en la sección anterior. 


\subsection{Configuración intensional}

Si un niño realiza correctamente la siguiente tarea:

Tarea 1: Pinta de color rojo los triángulos, de verde los círculos (redondos), de azul los cuadrados, de amarillo los rectángulos y de negro los rombos,

podemos afirmar que ha generalizado o abstraído aspectos figurativos de los conceptos generales de triángulo, círculo, cuadrado, rectángulo y rombo, y los está aplicando al caso particular de los dibujos que se le presentan.

Asimismo, el niño que responde a la pregunta, ¿Cuántas canicas tienes?, mostrando cinco dedos, pronunciando la palabra cinco, dibujando cinco palotes, o escribiendo el símbolo 5, ha realizado un proceso de generalización o abstracción, por lo que podríamos decir que ha alcanzado un cierto nivel de abstracción. Ciertamente que aún puede que no sea capaz de relacionar y operar con tales objetos intensivos usando el recurso de los símbolos numéricos, pero no se puede negar que ha desarrollado una cierta capacidad de generalización. Un primer grado de algebrización se debe reconocer, por tanto, asociado a la presencia de objetos intensivos (configuración intensional). Si adoptamos una visión más restringida del pensamiento algebraico, como la descrita por Radford, esta configuración intensional no sería considerada de índole algebraica, no tanto porque no se use notación y cálculo algebraico, sino porque el sujeto no genera la regla que describe el caso general.

No es necesario representar con símbolos literales los objetos intensivos para que dichos objetos intervengan en una práctica matemática. El uso de símbolos literales será necesario, o al menos, de gran utilidad para representar intensivos de mayor nivel de generalidad. Por ejemplo, el número 428 es una forma eficiente de representar cuatro centenas, dos decenas y 8 unidades, esto es, $4 \times 100+2 \times 10+8$. Si esta expresión se presenta a los estudiantes como un ejemplo de la expresión más general, $a \times 10^{2}+b \times 10+c$, estamos introduciéndoles en un primer nivel de razonamiento algebraico. A su vez, la expresión polinómica, $a \times 10^{2}+b \times 10+c$, se puede presentar como un caso particular de la expresión polinómica general de cualquier número en base 10, o en otra base diferente. También se puede generalizar al caso en que las potencias de la base sean negativas, esto es, para representar números decimales. 


\subsection{Configuración relacional}

Veamos el problema 2, mencionado en la primera sección del trabajo, resuelto de dos maneras diferentes. En ambos casos se moviliza una configuración de tipo relacional, pero la primera solución se puede calificar de más algebraica:

Problema 2: Tres amigos, Pedro, Antonio y Pablo, no se ponen de acuerdo sobre su edad. Pedro es más viejo que Pablo; Pablo es más joven que Antonio; Antonio, a su vez, es más viejo que Pedro. ¿Quién tiene más edad?, ¿Y menos?

Solución 1: La Figura 4 muestra la solución dada por un niño.

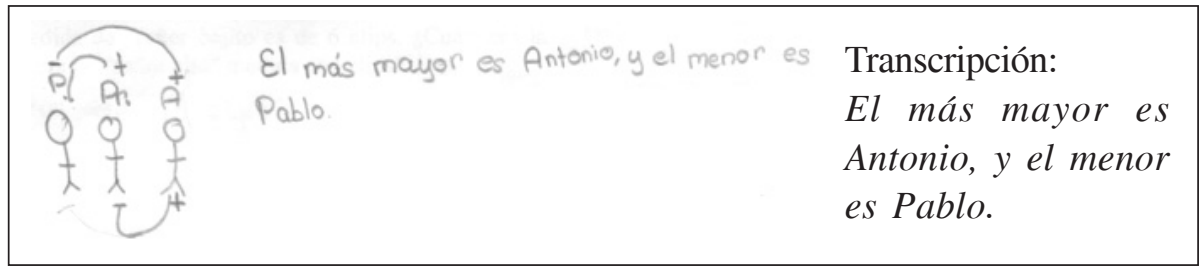

Figura 4 - Solución del problema de las edades

En esta resolución podemos reconocer rasgos de razonamiento algebraico de tipo relacional, según la definición dada. Las edades de Pedro, Antonio y Pablo son desconocidas; sus valores pueden variar dentro de un rango. El conjunto de valores posibles de cada una de las edades es un objeto intensivo. Entre las edades hay relaciones de desigualdad; la comparación de las edades requiere poner en juego la propiedad transitiva de la relación de orden en el conjunto numérico de los naturales aplicada a conjuntos de valores. Se aprecia el uso de recursos gráficos- un arco- para vincular las edades que se comparan, y el uso de los símbolos más y menos para indicar mayor y menor respectivamente.

Solución 2: Pedro es más viejo que Pablo, por ejemplo, Pedro tiene 15 años y Pablo 12. Antonio es más viejo que Pedro, por ejemplo, 16 años. O sea, Pablo es el más joven y Antonio el más viejo.

Esta solución se basa en valores particulares dados a las edades; son objetos extensivos. No obstante, esta solución también requiere movilizar una propiedad algebraica, la transitividad de la relación de orden en $\mathrm{N}$, aquí particularizada en la comparación de los tres números, 12, 15 y 16. El modo de razonamiento de la solución 1 se puede considerar más algebraico que el de la solución 2 al poner en juego más cantidad de objetos intensivos y el esbozo de una notación simbólica. 


\subsection{Configuración operacional}

El problema 1, incluido en la primera sección, es un ejemplo de tarea que pone en juego una configuración de tipo operacional. Se utilizan letras para representar las incógnitas, las relaciones se establecen mediante una ecuación, se opera con las incógnitas aplicando las definiciones y propiedades de las operaciones aritméticas.

Se puede calificar de configuración operacional, aplicativa (se aplican los conceptos de incógnita y ecuación, el conjunto de valores posibles de las incógnitas), implicando la transformación del enunciado dado en lenguaje natural a lenguaje alfanumérico.

\subsection{Configuración relacional- operacional}

Tarea 2: ¿Qué número hay que poner en lugar de [ ] en la expresión, 67 $+83=[]+82$ ?

Solución: Un alumno puede resolver la tarea sumando y restando 1 al primer miembro de la igualdad, $67+1+83-1$, obtiene $68+82$; a continuación resta 82 a ambos miembros y obtiene [ ] $=68$.

De esta manera, aplica propiedades generales de la relación de equivalencia y la propiedad asociativa de la adición.

Este modo de pensar y de resolver tareas con expresiones numéricas se conoce en la bibliografía sobre early algebra como características del pensamiento relacional ${ }^{3}$. Esto no tiene lugar si un alumno realiza la suma del primer término y después resta 82 ; obtiene el resultado 68, pero en este caso pone en juego hechos numéricos particulares.

Se trata de una configuración de tipo relacional - operacional, aplicativa, expresada con lenguaje ordinario y numérico.

Tarea 3: Encuentra los valores que hacen cada una de las siguientes sentencias numéricas verdaderas: $44+29=45+a ; 65+38=62+$ b; $99+87$ $=98+86+\mathrm{c}$.

Este ejemplo pone en juego también una configuración de tipo mixto, relacional y operacional, pero introduciendo el uso de notación simbólica literal.

\footnotetext{
${ }^{3}$ Se trata de un uso excesivamente restrictivo de la expresión pensamiento relacional al tratarse sólo de tareas que involucran el uso de números y operaciones aritméticas. La idea de comprensión relacional de Skemp (1976) puede estar en la base del uso de esta caracterización de las actividades algebraicas elementales, la cual se aplica al aprendizaje de cualquier contenido matemático.
} 


\subsection{Configuración funcional}

El concepto central es el de función, vinculado a un patrón que se expresa gráficamente, pero que puede ser expresado usando otros objetos ostensivos. En la solución a la tarea 4 se pueden reconocer los conceptos de variación, variable independiente, variable dependiente.

Tarea 4: Una bacteria se reproduce por reproducción celular. De cada una se obtienen dos. ¿Cuántas bacterias formarán parte de la cuarta generación? ¿Y en la quinta generación? ¿Y en la generación número 100?

Se establece una dependencia funcional entre la generación (variable independiente) y el número de bacterias correspondiente (variable dependiente). El lenguaje es aritmético y se pretende generar la regla general, o criterio de la correspondencia, al cual se puede llegar por multiplicaciones sucesivas y, subsecuentemente, por el reconocimiento del uso de potencias. Lo que podría desembocar en la expresión funcional que establece que a la generación $n$ le corresponde $2^{\mathrm{n}}$ bacterias.

Calificamos esta configuración como de tipo funcional, generativo (se debe reconocer el criterio general de la correspondencia), expresada con lenguaje natural y numérico.

\subsection{Configuración estructural}

Intervienen como objetos centrales las propiedades estructurales de las operaciones.

En libros de primaria encontramos elementos teóricos que suponen el inicio de una reflexión sobre la estructura algebraica de los conjuntos y operaciones con números. Tal es el caso de los enunciados generales de las propiedades conmutativa, asociativa y distributiva de las operaciones aritméticas y su aplicación a la solución de problemas, como en Ferrero (1999). 


\begin{tabular}{|c|c|c|}
\hline \multicolumn{3}{|l|}{ Las propiedades de la suma } \\
\hline $\begin{array}{l}\text { Propiedad conmutativa } \\
\text { El orden de los sumandos no altera la suma }\end{array}$ & \multicolumn{2}{|c|}{$\begin{array}{l}\text { Propiedad asociativa } \\
\text { Para sumar tres números, sumamos dos } \\
\text { cualesquiera de ellos y el resultado se suma con el } \\
\text { tercero. }\end{array}$} \\
\hline \multicolumn{3}{|l|}{ Relaciones entre los términos de la resta } \\
\hline \multicolumn{2}{|c|}{$\begin{array}{l}\text { Para comprobar si una resta está bien hecha se } \\
\text { suma el sustraendo con la diferencia y el resultado } \\
\text { debe ser el minuendo }\end{array}$} & $\begin{array}{l}M-S=D \\
S+D=M \\
M-D=S\end{array}$ \\
\hline \multicolumn{2}{|c|}{$\begin{array}{l}\text { En una resta, la diferencia no varía cuando se } \\
\text { suma o se resta un mismo número al minuendo y al } \\
\text { sustraendo }\end{array}$} & \\
\hline \multicolumn{3}{|c|}{ Propiedades conmutativa y asociativa de la multiplicación } \\
\hline $\begin{array}{l}\text { Propiedad conmutativa } \\
\text { En una multiplicación, el orden de los } \\
\text { factores no altera el resultado }\end{array}$ & \multicolumn{2}{|c|}{\begin{tabular}{|} 
Propiedad asociativa \\
Para multiplicar tres números, se multiplican \\
primero dos de ellos y el resultado por el tercero
\end{tabular}} \\
\hline \multicolumn{3}{|l|}{ Propiedad distributiva } \\
\hline $\begin{array}{l}\text { El producto de una suma por un número es } \\
\text { igual a la suma de los productos de cada sumando } \\
\text { por ese número }\end{array}$ & \multicolumn{2}{|c|}{$\begin{array}{l}\text { El producto de una diferencia por un número es } \\
\text { igual a la diferencia de los productos de cada término por } \\
\text { ese número }\end{array}$} \\
\hline
\end{tabular}

Cuadro 1 - Propiedades estructurales de $\mathrm{N}(+, \mathrm{x})$

\section{Síntesis y reflexiones finales}

La clarificación de la naturaleza de la práctica matemática en sus diversas áreas de contenido, y de los objetos y procesos que intervienen en la misma, es una cuestión de interés para la investigación en educación matemática. Esto es así porque la educación se ocupa de mejorar la enseñanza y el aprendizaje y un paso previo deberá ser comprender con profundidad los conocimientos y competencias que se desean promover y desarrollar. En este trabajo hemos abordado esta problemática para el caso del álgebra, rama de las matemáticas que sirve de herramienta de trabajo para los restantes campos de la matemática, así como área de investigación en sí misma, y que la investigación didáctica reconoce como particularmente conflictiva para los estudiantes.

Distintos autores han tratado de explicitar los rasgos característicos del álgebra, habiendo un cierto consenso en destacar la generalización como un proceso clave de la misma, y por tanto la noción de variable, ya que como afirma Dörfler (1991, p. 84), "generalizar significa construir variables". Otro rasgo característico del álgebra es el tratamiento de situaciones en las cuales intervienen cantidades o valores indeterminados, esto es, el uso de incógnitas y ecuaciones que modelizan dichas situaciones y que, mediante el cálculo (algebraico), se da respuesta a las mismas. Tanto para las situaciones que requieren generalización como para el manejo de las indeterminadas se utiliza 
una forma analítica de expresión característica y eficaz, usualmente alfanumérica.

Las investigaciones sobre early algebra han puesto, además, el acento en otros aspectos del razonamiento algebraico elemental, en particular, las situaciones de índole relacional, en principio no reducibles a las situaciones de generalización ni indeterminación, así como al uso, con frecuencia implícito, de ciertas propiedades estructurales de los sistemas numéricos. Además, se reconoce que las situaciones y prácticas algebraicas pueden implementarse apoyadas en el uso de la lengua natural, y otras formas no analíticas de expresión.

En este trabajo hemos tratado de elaborar un modo de ver la práctica algebraica, y el pensamiento que la acompaña, desde una perspectiva global. Para ello hemos aplicado algunas nociones del enfoque ontosemiótico, el cual mediante la adopción de supuestos pragmatistas, antropológicos y semióticos sobre el conocimiento matemático permite tener en cuenta los diversos objetos, procesos y facetas que intervienen en la actividad matemática.

Aunque no podamos dar una respuesta definitiva al problema de caracterización del álgebra, y que será necesario profundizar en la reflexión y el debate, nos parece que la dualidad extensivo-intensivo puede servir de base para dar cuenta de tres rasgos característicos del álgebra,

- la indeterminación, uso de incógnitas, ecuaciones y nociones relacionadas

- la generalización, uso de variables, fórmulas, parámetros

- la relación, binaria o de otro tipo

En estos tres tipos de situaciones o tareas matemáticas se puede reconocer la participación de objetos intensivos, esto es, conjuntos o clases de elementos agrupados mediante la intervención de un criterio o regla. El reconocimiento de dicha regla por el sujeto que realiza la actividad se considera como un requisito necesario, por algunos autores, para que dicha actividad se considere como algebraica y no simplemente como conceptualización.

Parece necesario distinguir entre situaciones de generalización e indeterminación; en el primer caso, se trata de construir un intensivo, encontrando un modo de expresar un elemento cualquiera del conjunto o clase de elementos que se deben considerar como un todo unitario. En el segundo se supone dado un objeto intensivo y la tarea consiste en hallar un elemento particular, fijo, pero indeterminado. Ambos casos son considerados, en nuestro modelo, dentro del tipo de configuración intensional, en un caso generativa, y en otro aplicativa.

Otra noción que puede ayudar a caracterizar las prácticas matemáticas de índole algebraica es la de configuración algebraica y sus tipos, entendida 
como el sistema semiótico formada por la red de objetos y procesos que intervienen en la solución de las tareas sobre las cuales se centran las prácticas (GODINO et al., 2011).

La consideración simultánea de los grados de intensión de los objetos que intervienen en una práctica y del tipo de lenguajes que se usan, analítico alfanumérico, versus icónico, gestual, o natural, permite definir grados de algebrización, lo cual puede ayudar a superar la brecha o ruptura mediante la cual se describe, con frecuencia, la práctica algebraica que se realiza en educación secundaria, frente al trabajo matemático que se realiza en educación primaria.

El razonamiento algebraico, entendido como hemos descrito en este trabajo, se reconoce presente en muchas tareas del currículo matemático de la escuela primaria. El álgebra es una forma de pensar y actuar en matemáticas caracterizada esencialmente por la dialéctica entre los procesos de generalización - particularización, y, en consecuencia, por la intervención y emergencia de objetos intensivos de niveles progresivos de generalidad. Los procesos de algebrización no solamente se pueden aplicar a tareas propias de la aritmética, sino pertenecientes también a la medida, la geometría, y el análisis de datos. El álgebra es más que un instrumento de modelización y más que un lenguaje simbólico; es una forma de pensar y actuar en matemáticas, una actitud a generalizar, y, por tanto, a simbolizar y operar con símbolos, que penetra todas sus ramas y las impulsa hacia nuevos niveles de creatividad.

Nuestras reflexiones sobre la naturaleza del razonamiento algebraico tienen fuertes implicaciones para la formación de profesores de matemáticas. Si queremos que dicho razonamiento penetre en las aulas de primaria, y mejorar el tratamiento del álgebra en secundaria, el profesor debe ser el principal agente del cambio. No basta con elaborar propuestas curriculares (NCTM, 2000) que incluyan el álgebra desde los primeros niveles educativos. Es necesario que los profesores participen de la visión ampliada del álgebra que hemos descrito en este trabajo a fin de que estén capacitados para transformar las tareas matemáticas escolares hacia el logro de niveles progresivos de algebrización.

\section{Reconocimiento:}

Trabajo realizado en el marco del proyecto de investigación, EDU201014947, Ministerio de Ciencia e Innovación (MICINN) y fondos FEDER. 


\section{Referencias}

ARZARELLO, F. Semiosis as a multimodal process. Revista Latinoamericana de Investigación en Matemática Educativa, México, D.F., v. 9, Número especial, p. 267 299. mar. 2006.

BEDNARZ, N. Emergence and development of algebra as problem solving tool: Continuities and discontinuities with arithmetic. In: BEDNARZ, N.; KIERAN, C.; LEE, L. (Eds.), Approaches to Algebra. Dordrecht: Kluwer Academic Publishers, 1996, p. 115 $-136$.

BEDNARZ, N.; KIERAN, C.; LEE, L. Approaches to Algebra: perpectives for Research and Teaching. Dordrecht: Kluwer Academic Publishers, 1996.

BOLEA, P.; BOSCH, M.; GASCÓN, J. La transposición didáctica de organizaciones matemáticas en proceso de algebrización. El caso de la proporcionalidad. Recherches en Didactique des Mathématiques, Grenoble, v. 2, n. 3, p. 247 -304, 2001.

CAI, J.; KNUTH, E. Early Algebraization. A global dialogue from multiple perspectives. Berlin: Springer-Verlag, 2011.

CARPENTER, T. P.; FRANKLE, M. L. ; LEVI, L. Thinking Mathematically. Integrating Arithmetic and Algebra in Elementary School. Portsmouth, NH: Heinemann, 2003.

CARPENTER, T.; LEVI, L.; FRANKE, M.L.; ZERINGUE, J.K. Algebra in elementary school: Developing relational thinking. ZDM. The International Journal on Mathematics Education, Berlin, v. 37, n. 1, p. 53 - 59, 2005.

CARRAHER, D. W.; MARTÍNEZ, M. V.; SCHLIEMANN. A. D. Early algebra and mathematical generalization. ZDM The International Journal on Mathematics Education, Berlin, v. 40, n. 1, p. 3 - 22, 2008.

CARRAHER, D. W.; SCHLIEMANN, A. L. Early algebra and algebraic reasoning. In: LESTER, F. (Ed.) Second Handbook of Research on Mathematics Teaching and Learning. Charlotte, N.C: Information Age Publishing, Inc. y NCTM, 2007, v. 2, p. 669 -705 .

CHEVALLARD, Y. La transposición didáctica. Buenos Aires: Editorial Aique, 1991.

COOPER, T. J.; WARREN, E. The effect of different representations on years 3 to 5 students' ability to generalize. ZDM The International Journal on Mathematics Education, Berlin, v. 40, n. 1, p. 23 - 37, 2008. 
DAVIS, R. ICME-5 Report: Algebraic thinking in the early grades. Journal of Mathematical Behaviour, v. 4, n. 2, p. 195 - 208, 1985.

DÖRFLER, W. Forms and means of generalization in mathematics. In: BISHOP, A. J. et al. (Ed.) Mathematical Knowledge: Its Growth Throught Teaching. Dordrecht: Kluwer A.P, 1991, p. 63 - 85.

DUVAL, R. Eight problems for a semiotic approach in mathematics education. In: RADFORD, L.; SCHUBRING, G.; SEEGER, F. (Eds.) Semiotics in Mathematics

Education: Epistemology, History, Classroom, and Culture. Rotterdam: Sense Publishers, 2008. p. 39 - 62.

ENGLISH, L. D.; WARREN, E. Introducing the variable through pattern exploration. The Mathematics Teacher, v. 91, n. 2, p. 166 - 171, 1998.

FERRERO, L. Matemáticas 5. Serie Sol y Luna. Madrid: Anaya. 1999.

FILLOY, E.; ROJANO, T.; PUIG, L. Educational algebra: A theoretical and empirical approach. Berlin: Springer. 2008.

GODINO, J. D. Un enfoque ontológico y semiótico de la cognición matemática.

Recherches en Didactiques des Mathematiques, Grenoble, v. 22, n. 2/3, p. 237 - 284, 2002.

GODINO, J. D.; BATANERO, C. Significado institucional y personal de los objetos matemáticos. Recherche en Didactique des Mathématiques, Grenoble, v. 14, n. 3, p. 325 - 355, 1994.

GODINO, J. D.; BATANERO, C.; FONT, V. The onto-semiotic approach to research in mathematics education. ZDM. The International Journal on Mathematics Education, Berlin, v. 39, n. 1 - 2, p. 27 - 135, 2007.

GODINO, J. D.; FONT, V.; WILHELMI, M. R. LURDUY, O. Why is the learning of elementary arithmetic concepts difficult? Semiotic tools for understanding the nature of mathematical objects. Educational Studies in Mathematics, Berlin, v. 77, n. 2, p. 247 $-265,2011$.

KAPUT, J. Transforming algebra from a engine of inequity fo an engine of mathematical power by "algebrafying" the K-12 curriculum. Dartmouth, MA: National Center of Improving Student Learning and Achievement in Mathematics and Science, 2000. 
KAPUT, J.; BLANTON, M. L. Algebrafying the elementary mathematics experience. En: ICMI STUDY CONFERENCE, $12^{\text {th }}, 2001$, Melbourne, Austrália. Proceedings... Melbourne: University of Melbourne. 2001, v. 1, p. 344 - 350. (H. Chick, K. Stacey, J. Vicent., y J. Vicent (Eds). Artículo presentado en The Future of the Teaching and Learning of Algebra).

KAPUT, J.: CARRAHER, D. W.; BLANTON, M. L. Algebra in the early grades. New York: Routledge, 2008.

KIERAN, C. The early learning of algebra: A structural perspective. En: WAGNER, S.; KIERAN , C. (Eds.) Research issues in the learning of algebra. Reston, VA: NCTM y Lawrence Earlbaum, 1989. p. 163 - 171.

KIERAN, C. The learning and teaching of school algebra. In: GROUWS, D. (Ed.) Handbook of Research on Mathematics Teaching and Learning. New York: Macmillan, 1992. p. 390 - 419.

KIERAN, K. Learning and teaching algebra at the middle school through college levels. Building meaning for symbols and their manipulation. In: LESTER , F. (Ed.) Second Handbook of Research on Mathematics Teaching and Learning, Charlotte, N.C: Information Age Publishing, Inc. y NCTM, 2007, p. 707 - 762. vol. 2.

MASON, J. Expressing generality and roots of algebra. In: BEDNARZ, N.; KIERAN, C.; LEE, L. (Eds.) Approach to algebra: Perpectives for Research and Teaching. Dordrecht: Kluwer Academic Publisher, 1996. p. 65 - 86.

MASON, J.; PIMM, D. Generic examples: seeing the general in the particular. Educational Studies in Mathematics, Berlin, v. 15, n. 3, p. 277 - 289, 1984.

MOLINA, M.; CASTRO, E.; CASTRO, E. Elementary students' understanding of the equal sign in number sentences. Electronic Journal of Research in Educational Psychology, Almería, v.7, n. 1, p. 341 - 368, 2009. Disponible en: < http:// www.investigacion-psicopedagogica.org/revista/new/english/index.php?n=17 >. Acceso en: 9 marzo 2012.

NATIONAL COUNCIL OF TEACHERS OF MATHEMATICS. Principles and standards for school mathematics. Reston, VA: NCTM, 2000.

PUIG, L. Protoálgebra en Babilonia. Suma, Zaragoza, v.61, p. 93 - 98, jun. 2009.

RADFORD, L. Signs and meanings in students' emergent algebraic thinking: a semiotic analysis. Educational Studies in Mathematics, Berlin, v. 42, n. 3, p. 237 - 268, 2000. 
RADFORD, L. Gestures, speech, and the sprouting of signs: A semiotic-cultural approach to studentes' types of generalization. Mathematical Thinking and Learning, Vancouver, v. 5, n. 1, p 37 - 70, 2003.

RADFORD, L. Algebraic thinking from a cultural semiotic perspective. Research in Mathematics Education, Vancouver, v. 12, n. 1, p. 1 - 19, 2010.

RADFORD, L. Grade 2 Students' Non-Symbolic Algebraic Thinking. In: CAI, J.; KNUTH, E. (Eds.) Early Algebraization. A global dialogue from multiple perspectives. Berlin: Springer-Verlag, 2011.p. 303 - 322.

SKEMP, R. Relational understanding and instrumental understanding. Mathematics Teaching, Derby, Inglaterra, GB, v. 77, n. 3, p. 20 - 26, 1976.

STEPHENS, A. C. Equivalence and relational thinking: preservice elementary teachers' awareness of opportunities and misconceptions. Journal of Mathematics Teacher Education, Berlin, v. 9, n. 3, p. 249-278, 2006.

VERGNAUD, G. Long terme et court terme dans l'apprentissage de I' algebra. In: COLLOQUE FRANCO-ALLEMND DE DIDACTIQUE DES MATHEMATIQUES ET DE L' INFORMATIQUE, 1., 1988, Laborde, Paris. Actas... Paris: La Pensée Sauvage, 1988. p. $189-199$.

VERGNAUD, G. La théorie des champs conceptuels. Recherches en Didactiques des Mathématiques, Grenoble, v. 10, n. 2/3, p. 133 -170, 1990.

WAGNER, S.; KIERAN, C. An agenda for research on the learning and teaching of algebra. In: KIERAN, C.; WAGNER, S. (Eds.) Research Issues in the Learning and Teaching of Algebra. Reston, VA: NCTM-LEA, 1989. p. 220 - 237.

ZAZKIS, R.; LILJEDAHL, P. Generalization of patterns: The tension between algebraic thinking and algebraic notation. Educational Studies in Mathematics, Berlin, v. 49 , n. 3, p. $379-402,2002$.

Submetido em Abril de 2011. Aprovado em Julho de 2011. 


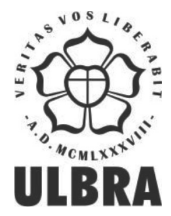

\section{ACTA SCIENTIAE}

Revista de Ensino de Ciências e Matemática
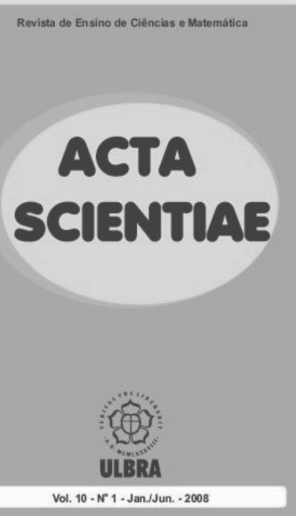

A Revista Acta Scientiae teve sua origem em 1999, mediante publicação de artigos oriundos dos pesquisadores das áreas de Ciências Naturais e Exatas da Universidade Luterana do Brasil - ULBRA Canoas (RS). Com sua indexação junto ao IBICT - Instituto Brasileiro de Informação em Ciência e Tecnologia (ISSN no. 1517-4492), é reconhecida como um espaço de publicação tanto de Ciências e Matemática como de Ensino dessas áreas. Entretanto, a partir do Volume 7, Número 1, 2005, Jan-Jun, a revista passa a publicar artigos exclusivos da área de Ensino de Ciências e Matemática, sendo editada desde sua fundação em dois números anuais. Assim, constitui-se em mais uma opção para publicação de artigos científicos dessa região de inquérito.

Confira:

http://www.ulbra.br/actascientiae

Você poderá realizar download dos exemplares da revista, encontrará informações para submissão e avaliação dos artigos.

\section{Atenção!}

A Revista Acta Scientiae é de fluxo contínuo para o recebimento de artigos. Além disso, ela é uma revista de divulgação impressa e online.

Informações:

mauriciomatematica@gmail.com

actascientiae@ulbra.br 